\title{
A solvent-free, base-catalyzed domino reaction towards trifluoromethylated benzenes from bio-based methyl coumalate
}

\begin{abstract}
Liang Chang, Nadja Klipfel, Luc Dechoux* and Serge Thorimbert*
A novel, efficient, and environmentally compatible method for $\mathrm{CF}_{3}$-substituted benzene production is reported. It sources a bio-based feedstock, employs $t \mathrm{BuOK}$ as catalyst, and is solvent-free. This regioselective approach provides various trifluoromethyl benzenes in good to excellent yields, without extra oxidant or special care. $\mathrm{CO}_{2}$ and water are the only byproducts of this process, and reaction conditions can scale up to gram quantities. The transformation involves an unprecedented $t \mathrm{BuOK}$-catalyzed domino process, and features Michael addition / 6 $\pi$-electrocyclic ring opening / [1,5]- $\mathrm{H}$ shift / carba-6 $\pi$-electrocyclic ring closure / decarboxylative aromatization reactions.
\end{abstract}

\section{Introduction}

$\mathrm{CF}_{3}$-functionalized aromatic and heterocyclic compounds play an essential role in medicinal chemistry, due to their unique pharmacokinetic properties. ${ }^{1}$ The increased lipophilicity and stability, brought by the $\mathrm{CF}_{3}$ group, improve bioavailability of the therapeutic compounds. Steric bulk introduced by the $\mathrm{CF}_{3}$ group may allow a better binding to target proteins. ${ }^{2}$ Thus, a wide range of methods have been described to introduce $\mathrm{CF}_{3}$ groups to aromatics. For instance, the metal-mediated coupling of $\mathrm{Ar}-\mathrm{H},{ }^{3} \mathrm{ArX}$ or $\mathrm{ArBX}_{2},{ }^{4}$ with $\mathrm{Si}-\mathrm{CF}_{3}$ or the TMG.ICF complex, ${ }^{5,6}$ hypervalent iodine, ${ }^{7}$ and by photoredox catalysis. ${ }^{8}$ The use of metals, large quantities of byproducts, and waste are major drawbacks to these approaches. There are atomeconomical alternatives for synthesis of trifluoromethylbenzenes. Namely, Cycloaddition (CA) reactions and formal-CA reactions which employ a "trifluoromethylated building block approach". For example, Co- and Rh-mediated [2+2+2] cycloaddition reactions with fluoroalkylated alkynes ${ }^{9}$, formal $[4+2]-C A$, and $[3+3]-C A$ have been reported ${ }^{10}$. The formal $[3+3]-C A$ between bis-silyl enol ethers and $\beta$-dimethyl thiounsaturated trifluoromethyl-ketones give tetrasubstituted arenes (Figure 1, a). ${ }^{10 \mathrm{~b}}$ The Diels-Alder (DA) cycloadditions between 1-ethoxy-3-trifluoromethyl-1, 3-butadiene, and electron-poor dienophiles yield trifluoromethyl-substituted benzenes (Figure 1, b). ${ }^{10 a}$ However, the number of studies reporting cycloadditions based on $\mathrm{CF}_{3}$ substrates is rather limited.

On the other hand, $a$-pyrones ${ }^{11}$ (e.g. methyl coumalate, MC) with dienophiles like alkynes, ${ }^{12}$ enolethers, ${ }^{13}$ or even simple alkenes $^{14}$, give substituted aromatics (methylbenzoates from MC). This happens through the Diels-Alder process after decarboxylation ${ }^{15}$ and eventually releases an alcohol molecule. As shown by Haufe and co-workers, $\mathrm{CF}_{3}$-substituted $\alpha$-pyrones react with electron-rich alkynes in an inverse electron-demand

Sorbonne Université, CNRS, Institut Parisien de Chimie Moléculaire, IPCM, F-75005 Paris, France.

† serge.thorimbert@sorbonne-universite.fr; luc.dechoux@sorbonne-universite.fr http://www.ipcm.fr/presentation-682?lang=en

Electronic Supplementary Information (ESI) available: [details of any supplementary information available should be included here]. See DOI: $10.1039 / \mathrm{x} 0 \times x \times 00000 \mathrm{x}$
Diels-Alder (IEDDA) reaction. This occurs under microwave irradiation, providing $\mathrm{CF}_{3}$-benzene in good yield (figure $1, \mathrm{c}$ ). ${ }^{12 \mathrm{c}}$

Figure 1. Approaches to trifluoromethylated benzene by cycloaddition reactions Although the Diels-Alder reaction remains exceptional in its scope and versatility for the methods of aromatic sixmembered rings architecture, the development of thermal carba- $6 \pi$-electrocyclization-aromatization process has come to complement [4+2] cycloadditions, when synthesizing polysubstituted benzene analogues. Funk and co-workers reported

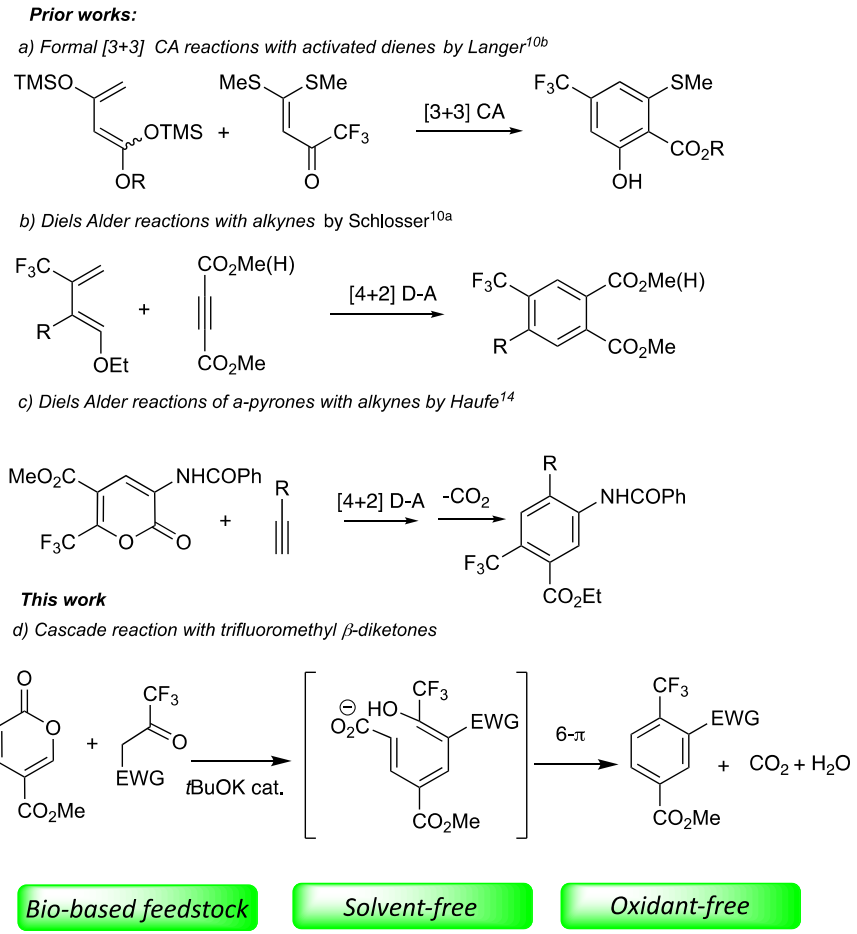

a streamlined electrocyclization-aromatization reaction of 2acyl-3-amino-hexatrienes. ${ }^{16}$ Anderson et al. developed a cascade cyclization of ynamides to azabicycles. ${ }^{17}$ Chuang et al. reported approaches to isatin derivatives, which utilize threecomponent reactions of phosphines, enynedioates, and cinnamaldimine. ${ }^{18}$ Recently, Huang et al. developed a new path to diaminobenzenes via highly electron-rich triene intermediates $^{19}$; however, the aromatization step of these procedures often required extra oxidizers. Furthermore, $6 \pi$ electrocyclization aromatization reactions for 
polyfunctionalized benzenes remain challenging. ${ }^{19}$ To the best of our knowledge, the synthesis of trifluoromethylated benzene via $6 \pi$-electrocyclization reaction has not been reported in previous literature.

Thus, considering the pharmacokinetic and pharmaceutical values of $\mathrm{CF}_{3}$-functionalized compounds we launched a program in devising an environmentally friendly synthetic method for the preparation of trifluoromethylated benzenes. In order to reach the key intermediate $\mathrm{CF}_{3}$-hexatriene (Figure $1, \mathrm{~d}$ ), MC has been selected as an electrophilic pro-diene synthon based on our recent finding that $\mathrm{MC}$ can be readily converted to diene in the presence of certain nucleophiles. ${ }^{20}$ Arising from dimerization of biorenewable malic acid, MC provides a basis for sustainable synthesis leading to novel bio-based product. We report herein a novel, environmentally compatible method towards $\mathrm{CF}_{3}$-substituted benzenes in solvent-free conditions.

\section{Results and discussion}

We began our study, by treating $\mathbf{M C}$ and trifluoroacetoacetophenone 1a with 1 equiv. of $\mathrm{Et}_{3} \mathrm{~N}$ in $\mathrm{DCM}$ at reflux for $30 \mathrm{~h}$. It provided the expected arene $2 \mathrm{a}$ in $54 \%$ yield (Table 1 , entry 1 ).

Optimization of the reaction conditions to obtain trifluoromethyl-arene $\mathbf{2} \mathbf{a}$ is presented in Table 1 . The solvent plays an important role in this type of transformation. Low conversions were observed in $\mathrm{THF}, \mathrm{EtOH}$, and water. This is due to trimerization of the starting diketone 1a, giving 1,3,6trifluoromethyl-2,4,5-triphenylbenzene (entries 2-4). Comparatively, toluene is the solvent of choice. It yields benzene $2 \mathrm{a}$ at $81 \%$ after $30 \mathrm{~h}$ at reflux, in the presence of stoichiometric amounts of $\mathrm{Et}_{3} \mathrm{~N}$ (entry 5). We found that simpler amines, such as the cyclic amidine 1,8-diazabicyclo[5.4.0]-undec-7-ene (DBU), are highly efficient and output $2 \mathbf{a}$ in $70 \%$ yields within an hour (entry 6 ).

To improve sustainability of the reaction conditions, we tested catalytic amounts of base. The quantity of DBU was successfully decreased to $10 \mathrm{~mol} \%$, providing product in $85 \%$ yield after $8 \mathrm{~h}$ (entry 7). However, separation of the expected product $\mathbf{2 a}$ and the starting diketone $\mathbf{1 a}$ was difficult. The reaction was extended to reach complete conversion and facilitate purification. After $15 \mathrm{~h}$ refluxing with 0.1 equiv. of $\mathrm{DBU}$, trifluoromethyl benzophenone 2a was isolated in $89 \%$ yield (entry 8). No improvement in this transformation was observed when 10 mol\% $t$ BuOK was employed (entry 9). Other bases such as Quinuclidine, DMAP, Imidazole, $\mathrm{Et}_{3} \mathrm{~N}$, or even DBN were not as efficient as DBU (see SI Table S1). Solventfree conditions led to the formation of expected compound $2 \mathrm{a}$ in $1.5 \mathrm{~h}$, but a moderate $67 \%$ yield (entry 10 ). Interestingly, the replacement of DBU by $t$ BuOK (10 mol\%) gave $2 \mathrm{a}$ in excellent yield (entry 11). Lowering the catalytic loading down to $5 \mathrm{~mol} \%$ resulted in a longer reaction time to reach similar yield (entry 12). Finally, a slight decrease in reaction temperature to $80^{\circ} \mathrm{C}$ enhance the isolated yield up to $95 \%$ (entry 13). No amelioration was observed with the inorganic bases $\mathrm{K}_{2} \mathrm{CO}_{3}$ or $\mathrm{KOH}$ (entry 14-15).
Table 1. Optimization of the Reaction Conditions ${ }^{a}$

\begin{tabular}{|c|c|c|c|c|}
\hline $\mathrm{CO}_{2} \mathrm{Me}$ & $\left.\underbrace{\mathrm{CF}_{3}}_{1 \mathbf{a}}\right|_{\mathrm{Ph}} ^{\mathrm{O}}$ & $\frac{\text { Base }}{\text { Ivent, ref }}$ & \multicolumn{2}{|c|}{$\mathrm{CO}_{2} \mathrm{Me} \mathbf{2 a}$} \\
\hline Entry & Base (equiv.) & Solvent & Time (h) & Yield $(\%)^{b}$ \\
\hline 1 & $\mathrm{Et}_{3} \mathrm{~N}(1)$ & DCM & 30 & 54 \\
\hline 2 & $\mathrm{Et}_{3} \mathrm{~N}(1)$ & THF & 30 & 29 \\
\hline 3 & $\mathrm{Et}_{3} \mathrm{~N}(1)$ & $\mathrm{EtOH}$ & 30 & 28 \\
\hline 4 & $\mathrm{Et}_{3} \mathrm{~N}(1)$ & $\mathrm{H}_{2} \mathrm{O}$ & 30 & ND \\
\hline 5 & $\mathrm{Et}_{3} \mathrm{~N}(1)$ & toluene & 30 & 81 \\
\hline 6 & DBU (1) & toluene & 1 & 70 \\
\hline 7 & DBU (0.1) & toluene & 8 & 85 \\
\hline 8 & DBU (0.1) & toluene & 15 & 89 \\
\hline 9 & tBuOK (0.1) & toluene & 15 & 80 \\
\hline 10 & $\mathrm{DBU}(0.1)$ & neat $^{c}$ & 1.5 & 67 \\
\hline 11 & tBuOK (0.1) & neat $^{c}$ & 1.5 & 90 \\
\hline 12 & tBuOK (0.05) & neat $^{c}$ & 4 & 93 \\
\hline 13 & tBuOK (0.1) & neat $^{d}$ & 2 & 95 \\
\hline 14 & $\mathrm{~K}_{2} \mathrm{CO}_{3}(0.1)$ & neat $^{c}$ & 1.5 & 74 \\
\hline 15 & $\mathrm{KOH}(0.1)$ & neat $^{c}$ & 1.5 & 70 \\
\hline
\end{tabular}

${ }^{\mathrm{a}}$ Reaction conditions: All reactions were performed with $\mathrm{MC}(1 \mathrm{mmol})$ and trifluoroaceto-acetophenone $1 \mathrm{a}(1 \mathrm{mmol}) .{ }^{\mathrm{b}}$ isolated yields. ${ }^{\mathrm{c}} \mathrm{At} 110^{\circ} \mathrm{C} .{ }^{\mathrm{d}} \mathrm{At} 80^{\circ} \mathrm{C}$.

With the optimal conditions established, we prepared various trifluoromethyl- $\beta$-diketones $\mathbf{1 a - t}$, according to known procedures. ${ }^{21}$ We then subjected each to the reaction conditions (MC, tBuOK $10 \mathrm{~mol} \%$, neat, $80^{\circ} \mathrm{C}$ ) (Table 2). Excellent regioselectivity is observed in the crude. ${ }^{19} \mathrm{~F}$ NMR spectroscopy shows $100 \%$ selectivity in favor of the benzophenone type product $\mathbf{2}$ vs its trifluoro-acetophenone regioisomer (ArCOCF3). Careful examination of the different spectra (See $\mathrm{SI}$ ) shows the $\mathrm{CF}_{3}$ group signal at around $-60 \mathrm{ppm}$. This is consistent with an aromatic $\mathrm{CF}_{3}$ moiety. ${ }^{22}$ The putative trifluoro-acetophenone regioisomer derivative would have its $\mathrm{COCF}_{3}$ signal shifted up-field at around $-70,-80 \mathrm{ppm}$. The effect of the substituent on the aromatic ring was investigated. Electron-neutral, electron-poor and electron-rich-groups were tolerated at the ortho-, meta- and para-positions. With the Methyl electron-donating groups, the corresponding benzenes 2b-d were formed in yields ranging from 90 to $96 \%$. Similarly, the methoxy p-electron-donating group provided expected trifluoromethyl arenes (2e-g) in yields up to $91 \%$. Halogensubstituted benzenes were obtained in $80-95 \%$ yields, no matter the halogen and its position on the aromatic ring $(\mathbf{2} \mathbf{h}-\mathbf{I})$. The ortho substituted nitro compound $\mathbf{2 0}$ was formed in $78 \%$ yield, whereas meta- and para- isomers (2m-n) were successfully isolated in $91-93 \%$ yield. 

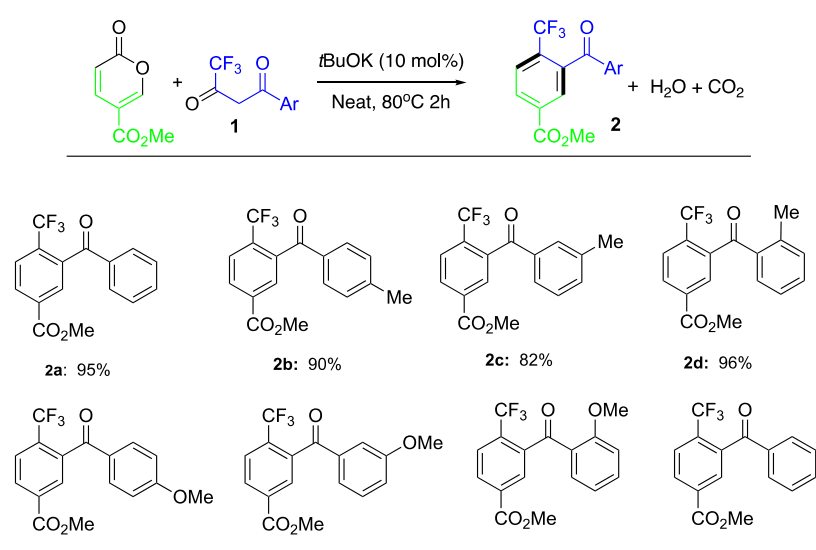

2b: $90 \%$

2c: $82 \%$

2d: $96 \%$
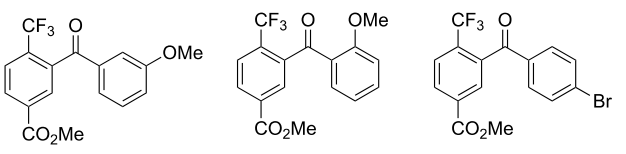

2e: $91 \%$

2f: $84 \%$

2g: $73 \%$

2h: $95 \%$
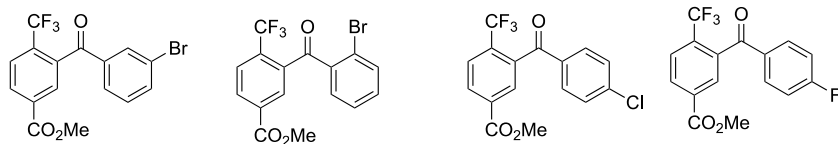

2i: $83 \%$

2j: $85 \%$
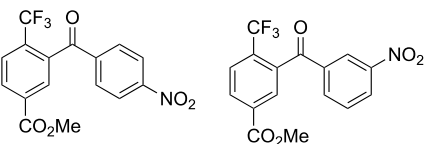

2k: $80 \%$

21: $93 \%$

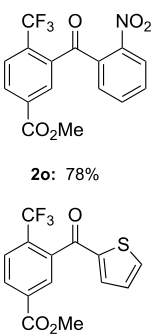

$\mathrm{CF}_{3}$

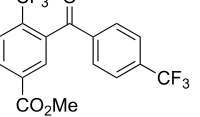

2m: $91 \%$

2n: $93 \%$

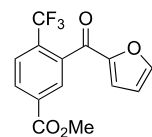

2r: $82 \%$

2s: $92 \%$

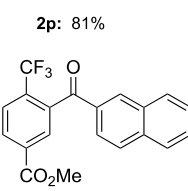

2t: $95 \%$

2q: $83 \%$
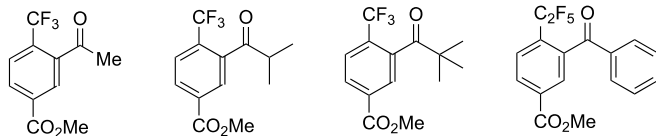

2u: $74 \% 2 h$

2w: NR 9h

2X: $71 \% 9 h$

The strongly electron-withdrawing trifluoromethyl group did not alter the efficiency of this domino reaction. It rendered the corresponding bis-trifluoromethyl derivatives $\mathbf{2 p - q}$, in yields up to $83 \%$. The reaction tolerates other aromatic rings like furan (2r, 82\%), thiophene (2s, 92\%), and naphthalene (2t, 95\%). In contrast to the previous results, reaction outcome is more erratic with trifluoro-alkyl diketones. Trifluoroaceto-acetone $\mathbf{1} \mathbf{u}$ gave the expected arene $\mathbf{2} \mathbf{u}$ in $76 \%$ yield, while the hindered diketone (1v; $\mathrm{R}=i-\mathrm{Pr})$ reacts slowly, and gives the product $\mathbf{2 v}$ in $58 \%$ yield after $9 \mathrm{~h}$. It is noteworthy that the more hindered diketone (1w; $\mathrm{R}=t-\mathrm{Bu})$ did not react in these conditions. The reaction could be extended to the pentafluoroethyl-acetophenone $\mathbf{1 x}$, which gave arene $\mathbf{2 x}$ in $84 \%$ yield. With the developed protocol in hand, trifluoromethyl acetophenone synthesis could be readily scaled up to gram quantities without difficulty, providing 1.87 g (89\%) of compound $\mathbf{2} \mathbf{b}$ after simple crystallization in $\mathrm{EtOH}$.

Figure 2. One-pot synthesis of $\mathrm{CF}_{3}$-functionalized fluorenone.

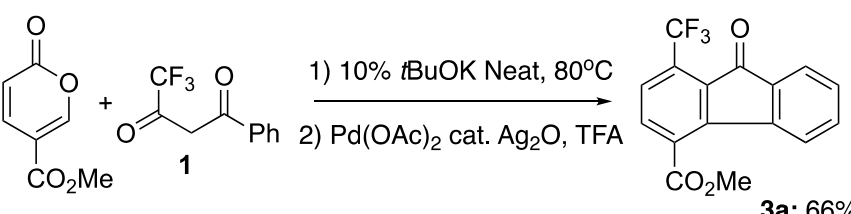

3a: $66 \%$

We also demonstrate that this reaction could incorporate with $\mathrm{Pd}$ catalyzed dehydrogenative cyclization, ${ }^{24}$ leading to $\mathrm{CF}_{3^{-}}$ functionalized fluorenone (3a, 66\%) in an efficient one-pot sequence.

Scheme 1. Plausible mechanism for the domino reaction leading to trifluoromethyl benzenes $\mathbf{2}$.

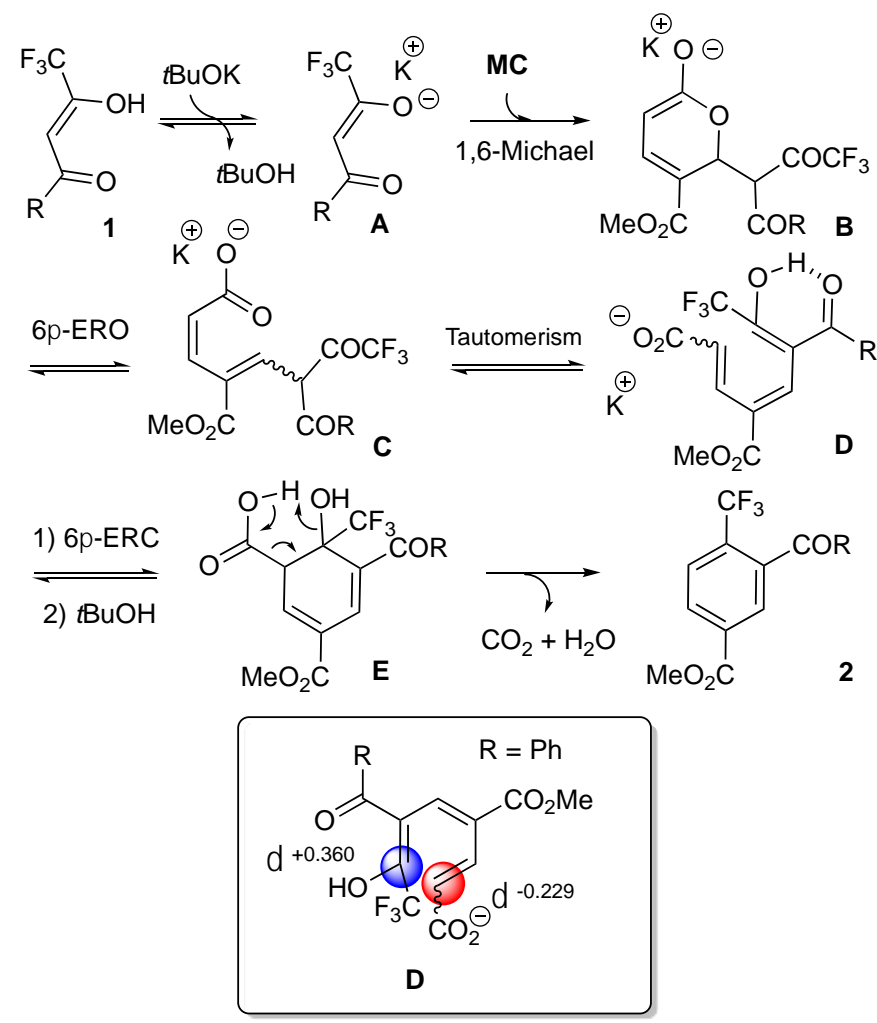

= calculated Hückel charge

To account for the generality of this method, a possible mechanism is presented in Scheme 1 . The base promoted the deprotonation of trifluoromethyl keto-enol $\mathbf{1}$ to release enolate $\mathbf{A}$. This enolate $\mathbf{A}$ adds to $\mathbf{M C}$ following a 1,6-Michael addition process, to generate the cyclic enolate B. $^{20}$ We propose that rapid $6 \pi$-electrocyclic ring opening $(6 \pi-E R O)$ of $\mathbf{B}$ leads to dienoate $\mathbf{C}^{20}$ The presence of the strong electron withdrawing $\mathrm{CF}_{3}$ group should favor the tauto-isomerization to carba-hexatriene $\mathbf{D}$. Carba- $6 \pi-E R C$ of hexatrienes is considered relatively disfavored; however, it is documented. ${ }^{23,26}$ The presence of an electron withdrawing group on the $\mathrm{C} 2$ position, as in intermediate $\mathbf{D}$, decreases the activation barrier energy by about $25 \mathrm{kcal} \mathrm{mol}^{-1}$. Moreover, the present case of $6 \pi-E R C$ might be facilitated by a push/pull type mechanism, between the hydrogen bonded enol and the proximal carbonyl (RCO). ${ }^{27}$ ChemDraw $^{\circledR}$ calculation of $\mathrm{CF}_{3}$-triene intermediate $\mathbf{D}$, applying the extended Hückel method, suggested both large partial positive and negative charges at $\mathrm{C}-1$ and $\mathrm{C}-6$. Carba-6 $\pi-$ electrocyclic ring closure would therefore be favored. ${ }^{23}$ Finally, 
the last step of the catalytic cycle involves a Doebner type ${ }^{28}$ decarboxylation-dehydration of intermediate $\mathbf{E}$ to give trifluoromethyl benzenes $\mathbf{2}$.

\section{Conclusions}

We have reported an efficient, solvent-free, and environmentally friendly reaction of methyl coumalate with trifluoromethyl- $\beta$-diketones, in a $t$ BuOK-catalyzed domino Michael $-6 \pi$-ERO - tautomerization $-6 \pi$-ERC - aromatization sequence. This procedure is a practical and sustainable approach, sourced from bio-renewable molecules (MC), for easy synthesis of myriad trifluoromethyl-benzene derivatives. Most bear the benzophenone core structure, ${ }^{29}$ which act on the biological targets of many diseases (e.g. cancer, Alzheimers, anti-viral and anti-bacterial drugs, prostatic hyperplasia, cardiovascular disease). ${ }^{30}$ In addition to broad substrate scope, this unprecedented synthetic approach has the advantages of readily available starting materials, lack of oxidizers, proceeds without a metallic catalyst, and releases only water and $\mathrm{CO}_{2}$. These soft experimental conditions do not need additive or special care (anhydrous or oxygen free conditions), and is applicable on the multi-gram scale.

\section{Experimental section}

\section{Materials and general methods}

All reactions were carried out under air with magnetic stirring. ${ }^{1} \mathrm{H}$ and ${ }^{13} \mathrm{C}$ NMR spectra were recorded at $400 \mathrm{MHz}$ for ${ }^{1} \mathrm{H}$ nuclei, $100 \mathrm{MHz}$ for ${ }^{13} \mathrm{C}$ nuclei. Chemical shifts are reported in $\delta$ units, parts per million (ppm) using, for ${ }^{1} \mathrm{H}$ and ${ }^{13} \mathrm{C}$, solvent residual peak as internal standard references: chloroform (7.26 ppm for ${ }^{1} \mathrm{H}$ NMR and $\left.77.16 \mathrm{ppm}\right)$. Coupling constants $(J)$ are given in $\mathrm{Hz}$, multiplicities are abbreviated as: $\mathrm{s}$ (singlet), bs (broad singlet), d (doublet), dd (doublet of doublets), ddd (doublet of doublets of doublets), $\mathrm{t}$ (triplet), $\mathrm{m}$ (multiplet). High resolution mass spectra (HR-MS) were recorded on a LTQOrbitrap Mass Spectrometer [Thermo Scientific]. IR: Shimadzu IRAffinity-1CE spectrometer, wavenumbers in $\mathrm{cm}^{-1}$.

Typical experimental procedure for the synthesis of compound 2 (a-w).

Methyl coumalate (154 mg, $1 \mathrm{mmol}, 1$ equiv), trifluoromethylB-diketone 1 ( $1 \mathrm{mmol}, 1$ equiv) and $t$ BuOK (11 mg, $0.1 \mathrm{mmol}$, 0.1 equiv) were mixed in a scklenk tube and stirred at $80{ }^{\circ} \mathrm{C}$ for 2 hours. The reaction mixture was cooled to room temperature, directly loaded to a plug of silica gel and eluted with cyclohexane/EtOAc (5/1 V/V). Evaporation of the solvent under vacuum delivered the expected products 2 .

\section{New compound characterization data}

Methyl 3-benzoyl-4-(trifluoromethyl) benzoate (2a): colorless oil (293 mg, 95\%); ${ }^{1} \mathbf{H}$ NMR (400 MHz, $\left.\mathrm{CDCl}_{3}\right) \delta 8.27$ (d, $J=8.2$ $\mathrm{Hz}, 1 \mathrm{H}), 8.05(\mathrm{~s}, 1 \mathrm{H}), 7.88(\mathrm{~d}, J=8.2 \mathrm{~Hz}, 1 \mathrm{H}), 7.77(\mathrm{~d}, J=7.4 \mathrm{~Hz}$, $2 \mathrm{H}), 7.63(\mathrm{t}, J=7.4 \mathrm{~Hz}, 1 \mathrm{H}), 7.48(\mathrm{t}, J=7.7 \mathrm{~Hz}, 2 \mathrm{H}), 3.94(\mathrm{~s}, 3 \mathrm{H})$; ${ }^{13} \mathrm{C}$ NMR $\left(100 \mathrm{MHz}, \mathrm{CDCl}_{3}\right) \delta 194.4,165.1,138.8(\mathrm{q}, J=2.0 \mathrm{~Hz})$, 136.0, 134.2, 133.1, 131.9 (q, $J=24.7 \mathrm{~Hz}), 130.7,130.2(2 \mathrm{C})$, 129.1, $128.7(2 \mathrm{C}), 127.1(\mathrm{q}, J=4.6 \mathrm{~Hz}), 123.1(\mathrm{q}, J=275.7 \mathrm{~Hz})$, 52.7; ${ }^{19}$ F NMR (376 $\left.\mathrm{MHz} \mathrm{CDCl}_{3}\right) \delta-58.5$; IR (film, $\mathrm{cm}^{-1}$ ): 2957,
1730, 1678, 1495, 1450, 1244, 988, 947, 754; HRMS (ESI) $m / z$ calcd for $\left[\mathrm{M}+\mathrm{Na}^{+}\right] \mathrm{C}_{16} \mathrm{H}_{11} \mathrm{~F}_{3} \mathrm{NaO}_{3} 331.0552$, found 331.0543.

Methyl 3-(4-methylbenzoyl)-4-(trifluoromethyl) benzoate (2b): yellow solid (291 mg, $90 \%$ ); mp: $106-107{ }^{\circ} \mathrm{C} ;{ }^{1} \mathbf{H}$ NMR $\left(400 \mathrm{MHz}, \mathrm{CDCl}_{3}\right) \delta 8.31-8.22(\mathrm{~m}, 1 \mathrm{H}), 8.06(\mathrm{~d}, J=0.6 \mathrm{~Hz}, 1 \mathrm{H})$, $7.88(\mathrm{~d}, J=8.2 \mathrm{~Hz}, 1 \mathrm{H}), 7.68(\mathrm{~d}, J=8.2 \mathrm{~Hz}, 2 \mathrm{H}), 7.28$ (d, $J=7.9$ $\mathrm{Hz}, 2 \mathrm{H}), 3.95(\mathrm{~s}, 3 \mathrm{H}), 2.44(\mathrm{~s}, 3 \mathrm{H}) ;{ }^{13} \mathrm{C}$ NMR $\left(100 \mathrm{MHz}, \mathrm{CDCl}_{3}\right) \delta$ $194.0,165.1,145.3,139.0$ (q, $J=2.0 \mathrm{~Hz}$ ), 133.5, 133.0, 131.8 $(q, J=32.7 \mathrm{~Hz}), 130.6,130.3,129.4,129.1,127.03$ (q, $J=4.6$ $\mathrm{Hz}), 123.14$ (q, $J=274.6 \mathrm{~Hz}$ ). 52.7, 21.7; ${ }^{19}$ F NMR (376 MHz, $\mathrm{CDCl}_{3}$ ) $\delta$-58.5; IR (film, cm ${ }^{-1}$ ): 2957, 1300, 1170, 1140, 1108; HRMS (ESI) $\mathrm{m} / z$ calcd for $\left[\mathrm{M}+\mathrm{Li}^{+}\right] \mathrm{C}_{17} \mathrm{H}_{13} \mathrm{~F}_{3} \mathrm{LiO}_{3} 329.0972$, found 329.0976 .

Methyl 3-(3-methylbenzoyl)-4-(trifluoromethyl) benzoate (2c): yellow solid (263 mg, $82 \%$ ); mp: $78-79{ }^{\circ} \mathrm{C}$; ${ }^{1} \mathbf{H}$ NMR (400 $\left.\mathrm{MHz}, \mathrm{CDCl}_{3}\right) \delta 8.43-8.21(\mathrm{~m}, 1 \mathrm{H}), 8.08(\mathrm{~d}, J=0.6 \mathrm{~Hz}, 1 \mathrm{H}), 7.90$ $(\mathrm{d}, J=8.2 \mathrm{~Hz}, 1 \mathrm{H}), 7.65(\mathrm{~s}, 1 \mathrm{H}), 7.54(\mathrm{~d}, J=7.6 \mathrm{~Hz}, 1 \mathrm{H}), 7.47(\mathrm{~d}, J$ $=7.5 \mathrm{~Hz}, 1 \mathrm{H}), 7.37(\mathrm{t}, J=7.5 \mathrm{~Hz}, 1 \mathrm{H}), 3.97(\mathrm{~s}, 3 \mathrm{H}), 2.42(\mathrm{~s}, 3 \mathrm{H})$; $\left.{ }^{13} \mathrm{C} \mathrm{NMR}\left(100 \mathrm{MHz}, \mathrm{CDCl}_{3}\right)\right) \delta 194.6,165.1,139.0(\mathrm{q}, J=2.0 \mathrm{~Hz})$, $138.7,137.7,136.0,135.0,133.1,131.8(q, J=32.7 \mathrm{~Hz}), 130.7$, $130.4,129.1,128.5,127.7,127.1(\mathrm{q}, J=4.6 \mathrm{~Hz}), 123.2(\mathrm{q}, J=$ $274.5 \mathrm{~Hz}), 52.7,21.2 ;{ }^{19} \mathrm{~F}$ NMR $\left(376 \mathrm{MHz}, \mathrm{CDCl}_{3}\right) \delta-58.5$; IR (film, cm ${ }^{-1}$ ): 1696, 1638, 1580, 1305, 1064,; HRMS (ESI) $\mathrm{m} / \mathrm{z}$ calcd for $\left[\mathrm{M}+\mathrm{Na}^{+}\right] \mathrm{C}_{17} \mathrm{H}_{13} \mathrm{~F}_{3} \mathrm{NaO}_{3} 345.0709,345.0721$.

Methyl 3-(2-methylbenzoyl)-4-(trifluoromethyl) benzoate (2d): yellow solid (309 mg, $96 \%$ ); mp: $99-100{ }^{\circ} \mathrm{C} ;{ }^{1} \mathbf{H}$ NMR (400 $\left.\mathrm{MHz}, \mathrm{CDCl}_{3}\right) \delta 8.20(\mathrm{~d}, J=8.2 \mathrm{~Hz}, 1 \mathrm{H}), 8.01(\mathrm{~s}, 1 \mathrm{H}), 7.82(\mathrm{~d}, J=$ $8.2 \mathrm{~Hz}, 1 \mathrm{H}), 7.39(\mathrm{t}, J=7.4 \mathrm{~Hz}, 1 \mathrm{H}), 7.29(\mathrm{~d}, J=7.6 \mathrm{~Hz}, 1 \mathrm{H}), 7.23$ (d, $J=6.8 \mathrm{~Hz}, 1 \mathrm{H}$ ), $7.14(\mathrm{t}, J=7.5 \mathrm{~Hz}, 1 \mathrm{H}), 3.88(\mathrm{~s}, 3 \mathrm{H}), 2.61(\mathrm{~s}$, $3 \mathrm{H}) ;{ }^{13} \mathrm{C}$ NMR $\left.\left(100 \mathrm{MHz}, \mathrm{CDCl}_{3}\right)\right) \delta 195.8,164.7,140.3,140.1$ (q, $J=1.8 \mathrm{~Hz}$ ), 135.1, 132.8, 132.5, 132.4, 131.9, 131.4 (q, $J=$ $32.0 \mathrm{~Hz}$ ), 130.5, 129.3, 126.8 (q, $J=4.7 \mathrm{~Hz}), 125.2,122.9$ (q, $J=$ $274.5 \mathrm{~Hz}), 52.3,21.2 ;{ }^{19} \mathrm{~F}$ NMR $\left(376 \mathrm{MHz}, \mathrm{CDCl}_{3}\right.$ ) $\delta-58.5$; IR (film, cm ${ }^{-1}$ ): 1702, 1623, 1371, 1263, 1193, 1124, 1026; HRMS (ESI) $\mathrm{m} / z$ calcd for $\left[\mathrm{M}+\mathrm{Na}^{+}\right] \mathrm{C}_{11} \mathrm{H}_{19} \mathrm{~F}_{3} \mathrm{NaO}_{3}$ 345.0709, found 345.0708 .

Methyl 3-(4-methoxybenzoyl)-4-(trifluoromethyl) benzoate (2e): yellow solid (308 mg, $91 \%$ ); mp: 108-109 ${ }^{\circ} \mathrm{C}$; ${ }^{1} \mathbf{H}$ NMR $\left(400 \mathrm{MHz}, \mathrm{CDCl}_{3}\right): \delta 8.20(\mathrm{~d}, J=8.2 \mathrm{~Hz}, 1 \mathrm{H}), 8.02(\mathrm{~s}, 1 \mathrm{H}), 7.82$ (d, $J=8.2 \mathrm{~Hz}, 1 \mathrm{H}), 7.70(\mathrm{~d}, J=8.9 \mathrm{~Hz}, 1 \mathrm{H}), 6.90(\mathrm{~d}, J=8.9 \mathrm{~Hz}$, $2 \mathrm{H}), 3.89(\mathrm{~s}, 3 \mathrm{H}), 3.82(\mathrm{~s}, 2 \mathrm{H}) ;{ }^{13} \mathrm{C}$ NMR $\left(100 \mathrm{MHz}, \mathrm{CDCl}_{3}\right): \delta$ 192.9, 165.1, 164.4, 133.0, $132.6(2 \mathrm{C}), 139.1$ (q, $J=2.0 \mathrm{~Hz}$ ), 131.6 (q, $J=32.6 \mathrm{~Hz}), 130.5,129.0,129.0,127.0$ (q, $J=4.5 \mathrm{~Hz}$ ), $123.2(q, J=274.6 \mathrm{~Hz}), 113.9(2 \mathrm{C}), 55.5,52.6 ;{ }^{19} \mathrm{~F}$ NMR (376 $\mathrm{MHz}, \mathrm{CDCl}_{3}$ ): $\delta-58.6$; IR (film, $\left.\mathrm{cm}^{-1}\right): 1690,1637,1587,1525$, 1287, 1212, 1183, 1146; HRMS (ESI) $\mathrm{m} / \mathrm{z}$ calcd for $\left[\mathrm{M}+\mathrm{Na}^{+}\right]$ $\mathrm{C}_{17} \mathrm{H}_{13} \mathrm{~F}_{3} \mathrm{NaO}_{4} 361.0658$, found 361.0664 .

Methyl 3-(3-methoxybenzoyl)-4-(trifluoromethyl) benzoate (2f): yellow solid (284 mg, $84 \%$ ); mp: $100-101{ }^{\circ} \mathrm{C} ;{ }^{1} \mathbf{H}$ NMR (400 $\mathrm{MHz}, \mathrm{CDCl}_{3}$ ): $\delta 8.24(\mathrm{~d}, J=8.2 \mathrm{~Hz}, 1 \mathrm{H}), 8.06(\mathrm{~s}, 1 \mathrm{H}), 7.85(\mathrm{~d}, J=$ $8.2 \mathrm{~Hz}, 1 \mathrm{H}), 7.44-7.39(\mathrm{~m}, 1 \mathrm{H}), 7.32(\mathrm{t}, J=7.9 \mathrm{~Hz}, 1 \mathrm{H}), 7.19(\mathrm{~d}$, $J=7.7 \mathrm{~Hz}, 1 \mathrm{H}), 7.14(\mathrm{dd}, J=8.2,2.6 \mathrm{~Hz}, 1 \mathrm{H}), 3.91(\mathrm{~s}, 3 \mathrm{H}), 3.81$ $(\mathrm{s}, 3 \mathrm{H}) ;{ }^{13} \mathrm{C}$ NMR $\left(100 \mathrm{MHz}, \mathrm{CDCl}_{3}\right): \delta 194.5,165.3,160.2,139.1$ (q, $J=1.9 \mathrm{~Hz}), 137.6,133.4,132.1(q, J=32.7 \mathrm{~Hz}), 131.1,129.9$, $129.4,127.4(q, J=4.5 \mathrm{~Hz}), 123.8,123.5(q, J=274.6 \mathrm{~Hz}$ ), 121.0, 114.1, 55.7, 53.0; ${ }^{19}$ F NMR (376 MHz, $\left.\mathrm{CDCl}_{3}\right)$ : $\delta$-58.6; IR (film, cm $\left.{ }^{-1}\right): 1693,1638,1589,1542,1378,1312,1289$, 
1258, 1214, 1183; HRMS (ESI): $\mathrm{m} / \mathrm{z}$ calcd for [M+K $\left.{ }^{+}\right]$ $\mathrm{C}_{17} \mathrm{H}_{13} \mathrm{~F}_{3} \mathrm{KO}_{4} 377.0398$, found 377.0388 .

Methyl 3-(2-methoxybenzoyl)-4-(trifluoromethyl) benzoate (2g): yellow solid (247 mg, $73 \%$ ); mp: 100-101 ${ }^{\circ} \mathrm{C} ;{ }^{1} \mathbf{H}$ NMR $\left(400 \mathrm{MHz}, \mathrm{CDCl}_{3}\right.$ ): $\delta 8.17(\mathrm{~d}, J=8.2 \mathrm{~Hz}, 1 \mathrm{H}), 7.95(\mathrm{~s}, 1 \mathrm{H}), 7.85-$ $7.74(\mathrm{~m}, 2 \mathrm{H}), 7.54$ (ddd, $J=8.5,7.4,1.8 \mathrm{~Hz}, 1 \mathrm{H}$ ), 7.05 (td, J = 7.4, $0.8 \mathrm{~Hz}, 1 \mathrm{H}), 6.91(\mathrm{~d}, J=8.3 \mathrm{~Hz}, 1 \mathrm{H}), 3.91(\mathrm{~s}, 3 \mathrm{H}), 3.51(\mathrm{~s}$, $3 \mathrm{H}) ;{ }^{13} \mathrm{C}$ NMR $\left(100 \mathrm{MHz}, \mathrm{CDCl}_{3}\right): \delta 193.5,165.2,159.4,142.0$ $(q, J=2.0 \mathrm{~Hz}), 135.2,132.7,131.6,130.5(\mathrm{q}, J=32.6 \mathrm{~Hz}), 129.8$, $127.9,126.4$ (q, $J=4.7 \mathrm{~Hz}), 125.8,123.1\left(\mathrm{CF}_{3}, \mathrm{q}, J=274.4 \mathrm{~Hz}\right)$, 120.5, 111.9, 55.2, 52.4; ${ }^{19} \mathrm{~F}$ NMR $\left(376 \mathrm{MHz} \mathrm{CDCl}_{3}\right): \delta-58.6$; IR (film, cm ${ }^{-1}$ ): 1693, 1626, 1543, 1318, 1295, 1271, 1208, 1162, 1143, 1067, 1028; HRMS (ESI) $\mathrm{m} / \mathrm{z}$ calcd for $\left[\mathrm{M}+\mathrm{Na}^{+}\right]$ $\mathrm{C}_{17} \mathrm{H}_{13} \mathrm{~F}_{3} \mathrm{NaO}_{4} 361.0658$, found 361.0665 .

Methyl 3-(4-bromobenzoyl)-4-(trifluoromethyl) benzoate (2h): yellow solid (366 mg, $95 \%$ ); mp: $76-77{ }^{\circ} \mathrm{C} ;{ }^{\mathbf{1}} \mathbf{H}$ NMR (400 $\left.\mathrm{MHz} \mathrm{CDCl}_{3}\right): \delta 8.27(\mathrm{~d}, J=8.2 \mathrm{~Hz}, 1 \mathrm{H}), 8.02(\mathrm{~s}, 1 \mathrm{H}), 7.87(\mathrm{~d}, J=$ $8.2 \quad \mathrm{~Hz}, \quad 1 \mathrm{H}), \quad 7.62(\mathrm{~m}, 4 \mathrm{H}), 3.90(\mathrm{~s}, 3 \mathrm{H})$; ${ }^{13} \mathrm{C}$ NMR $\left(100 \mathrm{MHz}, \mathrm{CDCl}_{3}\right): \delta$ 193.0, 164.6, $137.8(\mathrm{q}, J=2.0 \mathrm{~Hz})$, $133.7,134.5,132.9,131.8,131.5(q, J=32.7 \mathrm{~Hz}), 131.2,130.7$ 129.4, 128.7, 126.9 (q, $J=4.6 \mathrm{~Hz}), 122.8(\mathrm{q}, J=273.0 \mathrm{~Hz}), 52.5$; ${ }^{19}$ F NMR $\left(376 \mathrm{MHz}, \mathrm{CDCl}_{3}\right): \delta-58.4$; IR (film, $\left.\mathrm{cm}^{-1}\right): 1707$, $1628,1522,1260,1211,1185,1159,1127,1083$; HRMS (ESI): $\mathrm{m} / \mathrm{z}$ calcd for $\left[\mathrm{M}+\mathrm{Na}^{+}\right] \mathrm{C}_{16} \mathrm{H}_{10} \mathrm{BrF}_{3} \mathrm{NaO}_{3}$ 408.9658, found 408.9641 .

Methyl 3-(3-bromobenzoyl)-4-(trifluoromethyl) benzoate (2i): yellow solid (320 mg, $83 \%$ ); mp: 76-77 ${ }^{\circ} \mathrm{C} ;{ }^{1} \mathbf{H}$ NMR (400 MHz, $\left.\mathrm{CDCl}_{3}\right): \delta 8.28(\mathrm{~d}, J=8.2 \mathrm{~Hz}, 1 \mathrm{H}), 8.03(\mathrm{~s}, 1 \mathrm{H}), 7.92(\mathrm{t}, J=1.8 \mathrm{~Hz}$, $1 \mathrm{H}), 7.88(\mathrm{~d}, J=8.2 \mathrm{~Hz}, 1 \mathrm{H}$ ), 7.75 (ddd, $J=8.0,2.0,1.0 \mathrm{~Hz}, 1 \mathrm{H}$ ), $7.64(\mathrm{dt}, J=7.8,1.3 \mathrm{~Hz}, 1 \mathrm{H}), 7.35(\mathrm{t}, J=$ $7.9 \mathrm{~Hz}, 1 \mathrm{H}), 3.95(\mathrm{~s}, 3 \mathrm{H}) ;{ }^{13} \mathrm{C} \mathrm{NMR}\left(100 \mathrm{MHz}, \mathrm{CDCl}_{3}\right): \delta 193.3$, $165.2,138.2$ (q, $J=1.9 \mathrm{~Hz}$ ), 138.1, 137.3, 133.6, 132.9, 132.1 $(q, J=32.7 \mathrm{~Hz}), 131.4,130.6,129.3,129.2,127.6(q, J=4.5 \mathrm{~Hz})$, $123.4,123.4$ (q, $J=274.6 \mathrm{~Hz}), 53.1 ;{ }^{19}$ F NMR (376 MHz, $\mathrm{CDCl}_{3}$ ): $\delta-58.4$; IR (film, cm ${ }^{-1}$ ): 1701, 1607, 1275, 1209, 1162, 1145, 1120, 1075; HRMS (ESI): $\mathrm{m} / z$ calcd for $\left[\mathrm{M}+\mathrm{Na}^{+}\right] \mathrm{C}_{16} \mathrm{H}_{10} \mathrm{BrF}_{3} \mathrm{NaO}_{3}$ 408.9658, found 408.9622.

Methyl 3-(2-bromobenzoyl)-4-(trifluoromethyl) benzoate (2j): yellow solid (330 mg, $85 \%$ ); mp: $97-98{ }^{\circ} \mathrm{C} ;{ }^{1} \mathbf{H}$ NMR $(400 \mathrm{MHz}$, $\left.\mathrm{CDCl}_{3}\right): \delta 8.28(\mathrm{~d}, J=8.2 \mathrm{~Hz}, 1 \mathrm{H}), 8.03(\mathrm{~s}, 1 \mathrm{H}), 7.92(\mathrm{t}, J=1.8 \mathrm{~Hz}$, $1 \mathrm{H}), 7.88(\mathrm{~d}, J=8.2 \mathrm{~Hz}, 1 \mathrm{H}$ ), 7.75 (ddd, $J=8.0,2.0,1.0 \mathrm{~Hz}, 1 \mathrm{H}$ ), $7.64(\mathrm{dt}, J=7.8,1.3 \mathrm{~Hz}, 1 \mathrm{H}), 7.35(\mathrm{t}, J=$ $8.0 \mathrm{~Hz}, 1 \mathrm{H}), 3.95(\mathrm{~s}, 3 \mathrm{H}) ;{ }^{13} \mathrm{C} \mathrm{NMR}\left(100 \mathrm{MHz}, \mathrm{CDCl}_{3}\right): \delta 193.3$, $165.2,138.2$ (q, $J=1.9 \mathrm{~Hz}), 138.1,137.3,133.6,132.9,132.1$ $(q, J=32.7 \mathrm{~Hz}), 131.4,130.6,129.3,129.2,127.6(q, J=4.5 \mathrm{~Hz})$, $123.4,123.4$ (q, $J=274.6 \mathrm{~Hz}), 53.1 ;{ }^{19}$ F NMR (376 MHz, $\mathrm{CDCl}_{3}$ ): $\delta$-58.4; IR (film, cm ${ }^{-1}$ ): 1705, 1602, 1380, 1311, 1291, 1268, 1247, 1210, 1189, 1168, 1151, 1133, 1121; HRMS (ESI): $\mathrm{m} / \mathrm{z}$ calcd for $\left[\mathrm{M}+\mathrm{Na}^{+}\right] \mathrm{C}_{16} \mathrm{H}_{10} \mathrm{BrF}_{3} \mathrm{NaO}_{3} 408.9658$, found 408.9650 .

Methyl 3-(4-chlorobenzoyl)-4-(trifluoromethyl) benzoate (2k): yellow solid (273mg, 80\%); mp: $110-111{ }^{\circ} \mathrm{C} ;{ }^{1} \mathrm{H}$ NMR $(400 \mathrm{MHz}$, $\left.\mathrm{CDCl}_{3}\right) \delta 8.29(\mathrm{ddd}, J=8.2,1.6,0.8 \mathrm{~Hz}, 1 \mathrm{H}), 8.08-8.02(\mathrm{~m}, 1 \mathrm{H})$, $7.90(\mathrm{~d}, J=8.2 \mathrm{~Hz}, 1 \mathrm{H}), 7.76-7.67(\mathrm{~m}, 2 \mathrm{H}), 7.50-7.42(\mathrm{~m}, 2 \mathrm{H})$, $3.96(\mathrm{~s}, 3 \mathrm{H}) ;{ }^{13} \mathrm{C}$ NMR $\left(100 \mathrm{MHz}, \mathrm{CDCl}_{3}\right) \delta 193.1,164.9,140.8$, $138.2(q, J=2.0 \mathrm{~Hz}), 134.3,133.2,131.9(q, J=32.8 \mathrm{~Hz}), 131.5$, $131.0(2 \mathrm{C}), 127.2(\mathrm{q}, J=4.6 \mathrm{~Hz}), 129.1(2 \mathrm{C}), 129.0,123.0\left(\mathrm{CF}_{3}\right.$, $\mathrm{q}, J=274.6 \mathrm{~Hz}), 52.8 ;{ }^{19} \mathrm{~F}$ NMR $\left(376 \mathrm{MHz}, \mathrm{CDCl}_{3}\right) \delta-58.4$; IR (film, $\mathrm{cm}^{-1}$ ): 2955, 1734, 1673, 1587, 1439, 1168, 1043, 1011,
943, 916, 855, 780, 707; HRMS (ESI) $\mathrm{m} / \mathrm{z}$ calcd for [M+Na ${ }^{+}$] $\mathrm{C}_{16} \mathrm{H}_{10} \mathrm{ClF}_{3} \mathrm{NaO}_{3}$ 365.0163, found 365.0167.

Methyl 3-(4-fluorobenzoyl)-4-(trifluoromethyl) benzoate (2l): yellow solid (304mg, 93\%); mp: 95-96 ${ }^{\circ} \mathrm{C} ;{ }^{1} \mathrm{H}$ NMR $(400 \mathrm{MHz}$, $\mathrm{CDCl}_{3}$ ) $\delta 8.28(\mathrm{ddd}, J=8.2,1.6,0.8 \mathrm{~Hz}, 1 \mathrm{H}), 8.10-8.02(\mathrm{~m}, 1 \mathrm{H})$, $7.89(\mathrm{~d}, J=8.2 \mathrm{~Hz}, 1 \mathrm{H}), 7.86-7.76(\mathrm{~m}, 2 \mathrm{H}), 7.20-7.10(\mathrm{~m}, 2 \mathrm{H})$, $3.96(\mathrm{~s}, 3 \mathrm{H}) ;{ }^{13} \mathrm{C}$ NMR $\left(100 \mathrm{MHz}, \mathrm{CDCl}_{3}\right) \delta 192.8,166.3(\mathrm{~d}, J=$ $257.1 \mathrm{~Hz}), 165.0,138.4(\mathrm{q}, J=2.0 \mathrm{~Hz}) 133.2,132.9(\mathrm{~d}, J=9.7$ $\mathrm{Hz}), 131.8(\mathrm{q}, J=32.7 \mathrm{~Hz}) 130.9,129.0,127.16(\mathrm{q}, J=4.6 \mathrm{~Hz})$, $123.1(\mathrm{q}, J=274.6 \mathrm{~Hz}), 115.96(\mathrm{~d}, J=22.1 \mathrm{~Hz}) 52.8 ;{ }^{19} \mathrm{~F}$ NMR (376 $\left.\mathrm{MHz} \mathrm{CDCl}_{3}\right) \delta-58.50,-102.93(\mathrm{~m})$; IR (film, $\mathrm{cm}^{-1}$ ) : 2955, 1733, 1672, 1598, 1306, 1173, 1158, 1114, 1045, 983, 926, 850, 782, 715; HRMS (ESI) $\mathrm{m} / \mathrm{z}$ calcd for $\left[\mathrm{M}+\mathrm{Na}^{+}\right]$ $\mathrm{C}_{16} \mathrm{H}_{10} \mathrm{~F}_{4} \mathrm{NaO}_{3} 349.0458$, found 349.0465 .

Methyl 3-(4-nitrobenzoyl)-4-(trifluoromethyl) benzoate $(2 \mathrm{~m})$ : yellow solid (322 mg, 91\%); $\mathrm{mp}: 108-109{ }^{\circ} \mathrm{C}$; ${ }^{1} \mathbf{H}$ NMR (400 $\left.\mathrm{MHz}, \mathrm{CDCl}_{3}\right): \delta 8.32-8.30(\mathrm{~m}, 3 \mathrm{H}), 8.05(\mathrm{~s}, 1 \mathrm{H}), 7.96-7.89(\mathrm{~m}$, $3 \mathrm{H}), 3.91(\mathrm{~s}, 3 \mathrm{H}) ;{ }^{13} \mathrm{C}$ NMR (100 MHz, $\left.\mathrm{CDCl}_{3}\right): \delta 193.1,165.1$, $151.2,140.7,137.7,133.9,132.2(\mathrm{q}, J=32.9 \mathrm{~Hz}), 131.7(\mathrm{q}, J=$ $1.7 \mathrm{~Hz}$ ), 131.4, 129.3, 127.8 (q, $J=4.5 \mathrm{~Hz}$ ), $124.2(2 \mathrm{C}), 123.3$ (q, $J=274.5 \mathrm{~Hz}), 53.2 ;{ }^{19} \mathrm{~F}$ NMR $(376 \mathrm{MHz}, \mathrm{CDCl} 3): \delta-58.3$; IR (film, $\mathrm{cm}^{-1}$ ): 1732, 1681, 1530, 1306, 1270, 1252, 1171, 1148, 1115, 1044; HRMS (ESI): $\mathrm{m} / \mathrm{z}$ calcd for $\left[\mathrm{M}+\mathrm{Na}^{+}\right] \mathrm{C}_{16} \mathrm{H}_{10} \mathrm{~F}_{3} \mathrm{NNaO}_{5}$ 376.0403 , found 376.0393 .

Methyl 3-(3-nitrobenzoyl)-4-(trifluoromethyl) benzoate (2n): yellow solid (327 mg, 93\%); mp: $130-131{ }^{\circ} \mathrm{C}$; ${ }^{\mathbf{1}} \mathbf{H}$ NMR (400 $\left.\mathrm{MHz} \mathrm{CDCl}_{3}\right): \delta 8.54(\mathrm{t}, J=1.8 \mathrm{~Hz}, 1 \mathrm{H}), 8.48(\mathrm{dd}, J=8.2,1.6 \mathrm{~Hz}$, $1 \mathrm{H}), 8.33(\mathrm{dd}, J=8.3 \mathrm{~Hz}, 1 \mathrm{H}), 8.09(\mathrm{~d}, J=7.7 \mathrm{~Hz}, 1 \mathrm{H}), 8.06(\mathrm{~s}$, $1 \mathrm{H}), 7.93(\mathrm{~d}, J=8.2 \mathrm{~Hz}, 1 \mathrm{H}), 7.71(\mathrm{t}, J=7.7 \mathrm{~Hz}, 1 \mathrm{H}), 3.93(\mathrm{~s}, 3 \mathrm{H})$; ${ }^{13} \mathrm{C}$ NMR (100 MHz, $\left.\mathrm{CDCl}_{3}\right): \delta 191.9,164.5,148.1,137.1,136.9$ $(q, J=1.9 \mathrm{~Hz}), 135.1,133.3,131.6(q, J=32.8 \mathrm{~Hz}), 131.2,129.9$, $128.6,128.0,127.2$ (q, $J=4.5 \mathrm{~Hz}$ ), 124.3, 122.7 (q, $J=274.5$ $\mathrm{Hz}), 52.5 ;{ }^{19} \mathrm{~F}$ NMR $\left(376 \mathrm{MHz}, \mathrm{CDCl}_{3}\right): \delta-58.33$; IR (film, $\mathrm{cm}^{-1}$ ): $1732,1608,1581,1438,1350,1304,1269,1197,1158$; HRMS (ESI): $m / z$, calcd for $\left[\mathrm{M}+\mathrm{Na}^{+}\right] \mathrm{C}_{16} \mathrm{H}_{10} \mathrm{~F}_{3} \mathrm{NNaO}_{5} 376.0409$, found 376.0403 .

Methyl 3-(2-nitrobenzoyl)-4-(trifluoromethyl) benzoate (20): yellow solid (275 mg, $78 \%$ ); mp: $97-98{ }^{\circ} \mathrm{C} ;{ }^{1} \mathbf{H}$ NMR (400 MHz, $\left.\mathrm{CDCl}_{3}\right): \delta 8.26(\mathrm{~d}, J=8.2 \mathrm{~Hz}, 1 \mathrm{H}), 8.09-8.03(\mathrm{~m}, 1 \mathrm{H}), 8.01(\mathrm{~s}$, $1 \mathrm{H}), 7.93(\mathrm{~d}, J=8.2 \mathrm{~Hz}, 1 \mathrm{H}), 7.79-7.67(\mathrm{~m}, 2 \mathrm{H}), 7.57-7.50(\mathrm{~m}$, $1 \mathrm{H}), 3.90(\mathrm{~s}, 3 \mathrm{H}) ;{ }^{13} \mathrm{C} \mathrm{NMR}\left(100 \mathrm{MHz}, \mathrm{CDCl}_{3}\right): \delta 190.8,164.4$, $147.7,136.3$ (q, $J=1.4 \mathrm{~Hz}$ ), 134.0, 133.4, 133.1, 132.6, 132.5 (q, $J=33.3 \mathrm{~Hz}$ ), 132.1, 130.7, 130.2, 127.9 (q, $J=5.6 \mathrm{~Hz}$ ), 124.4, $122.6(q, J=274.5 \mathrm{~Hz}), 52.6 ;{ }^{19} \mathrm{~F} \mathrm{NMR}\left(376 \mathrm{MHz}, \mathrm{CDCl}_{3}\right): \delta-$ 59.0; IR (film, cm ${ }^{-1}$ ): 1715, 1630, 1561, 1488, 1333, 1305, 1285, 1211, 1121, 1070; HRMS (ESI): $\mathrm{m} / \mathrm{z}$ calcd for $\left[\mathrm{M}+\mathrm{Na}^{+}\right]$ $\mathrm{C}_{16} \mathrm{H}_{10} \mathrm{~F}_{3} \mathrm{NNaO}_{5}$ 376.0403, found 376.0415.

Methyl 3-(4-(trifluoromethyl)benzoyl)-4-(trifluoromethyl) benzoate (2p): yellow solid (304 mg, $81 \%$ ); mp: $78-79{ }^{\circ} \mathrm{C} ;{ }^{1} \mathbf{H}$ NMR $\left(400 \mathrm{MHz}, \mathrm{CDCl}_{3}\right): \delta 8.26(\mathrm{~d}, J=8.2 \mathrm{~Hz}, 1 \mathrm{H}), 8.09-8.03$ $(\mathrm{m}, 1 \mathrm{H}), 8.01(\mathrm{~s}, 1 \mathrm{H}), 7.93(\mathrm{~d}, J=8.2 \mathrm{~Hz}, 1 \mathrm{H}), 7.79-7.67(\mathrm{~m}$, $2 \mathrm{H}), \quad 7.57-7.50(\mathrm{~m}, \quad 1 \mathrm{H}), \quad 3.90 \quad(\mathrm{~s}, \quad 3 \mathrm{H})$; ${ }^{13} \mathrm{C}$ NMR $\left(100 \mathrm{MHz}, \mathrm{CDCl}_{3}\right): \delta 193.7,165.3,139.1,138.3$ (q, $J=$ $2.0 \mathrm{~Hz}), 135.8,135.7(q, J=32.8 \mathrm{~Hz}), 135.5,133.8,132.3(q, J=$ $32.8 \mathrm{~Hz}), 131.7,130.8,129.4,127.7(q, J=4.5 \mathrm{~Hz}), 126.2(\mathrm{q}, J=$ $3.7 \mathrm{~Hz}), 123.9\left(\mathrm{CF}_{3}, \mathrm{q}, J=272.9 \mathrm{~Hz}\right), 123.5(\mathrm{q}, J=274.5 \mathrm{~Hz})$, 53.2; ${ }^{19}$ F NMR (376 MHz, $\mathrm{CDCl}_{3}$ ): $\delta-58.48,-63.40$; IR (film, $\left.\mathrm{cm}^{-1}\right): 1732,1706,1602,1390,1284,1262,1214,1129,1085$, 
1054, 1027; HRMS (ESI): $\mathrm{m} / \mathrm{z}$ calcd for $\left[\mathrm{M}+\mathrm{Na}^{+}\right] \mathrm{C}_{17} \mathrm{H}_{10} \mathrm{~F}_{6} \mathrm{NaO}_{3}$ 399.0426, found 399.0427.

Methyl 3-(3-(trifluoromethyl)benzoyl)-4-(trifluoromethyl) benzoate (2q): yellow solid (312 mg, $83 \%$ ); mp: $71-72{ }^{\circ} \mathrm{C} ;{ }^{1} \mathbf{H}$ NMR $\left(400 \mathrm{MHz}, \mathrm{CDCl}_{3}\right): \delta 8.29(\mathrm{~d}, J=8.9 \mathrm{~Hz}, 1 \mathrm{H}), 8.06(\mathrm{~d}, J=6.4$ $\mathrm{Hz}, 2 \mathrm{H}), 7.97-7.82(\mathrm{~m}, 3 \mathrm{H}), 7.60(\mathrm{t}, J=7.8 \mathrm{~Hz}, 1 \mathrm{H}), 3.93(\mathrm{~s}$, $3 \mathrm{H}) ;{ }^{13} \mathrm{C}$ NMR $\left(100 \mathrm{MHz}, \mathrm{CDCl}_{3}\right): \delta 193.5,165.3,138.2$ (q, $J=1.9$ $\mathrm{Hz}), 137.0,133.9,133.8,132.7(q, J=32.8 \mathrm{~Hz}), 132.3(\mathrm{q}, J=23.2$ $\mathrm{Hz}), 131.7,130.9(\mathrm{q}, J=3.5 \mathrm{~Hz}), 129.9,129.4,127.8(\mathrm{q}, J=4.5$ $\mathrm{Hz}), 126.9(\mathrm{q}, J=3.8 \mathrm{~Hz}), 123.5\left(\mathrm{CF}_{3}, \mathrm{q}, J=274.5 \mathrm{~Hz}\right), 123.9(\mathrm{q}, J$ $=272.6 \mathrm{~Hz}), 53.2 ;{ }^{19} \mathrm{~F} \mathrm{NMR}\left(376 \mathrm{MHz}, \mathrm{CDCl}_{3}\right): \delta-58.44$, 63.01; IR (film, cm ${ }^{-1}$ ): 1732, 1706, 1602, 1390, 1284, 1262, 1214, 1129, 1085, 1054, 1027; HRMS (ESI): $\mathrm{m} / \mathrm{z}$ calcd for $\left[\mathrm{M}+\mathrm{Na}^{+}\right] \mathrm{C}_{17} \mathrm{H}_{10} \mathrm{~F}_{6} \mathrm{NaO}_{3} 399.0426$, found 399.0436.

Methyl 3-(furan-2-carbonyl)-4-(trifluoromethyl) benzoate (2r) : yellow solid (245 mg, $82 \%$ ); mp: $101-102{ }^{\circ} \mathrm{C} ;{ }^{1} \mathbf{H}$ NMR (400 $\left.\mathrm{MHz}, \mathrm{CDCl}_{3}\right) \delta 8.32-8.25(\mathrm{~m}, 1 \mathrm{H}), 8.18(\mathrm{~d}, J=0.6 \mathrm{~Hz}, 1 \mathrm{H}), 7.87$ $(\mathrm{d}, J=8.2 \mathrm{~Hz}, 1 \mathrm{H}), 7.07(\mathrm{~d}, J=3.3 \mathrm{~Hz}, 1 \mathrm{H}), 6.60$ (dd, $J=3.3,1.7$ $\mathrm{Hz}, 1 \mathrm{H}), 3.97(\mathrm{~s}, 3 \mathrm{H}) ;{ }^{13} \mathrm{C}$ NMR $\left(100 \mathrm{MHz}, \mathrm{CDCl}_{3}\right) \delta 181.2,165.0$, $151.7,148.4,137.4$ (q, $J=2.0 \mathrm{~Hz}), 133.1,132.0(\mathrm{q}, J=32.8 \mathrm{~Hz}$ ), 131.2, 129.4, 127.2 (q, $J=4.7 \mathrm{~Hz}), 123.0(q, J=275.1 \mathrm{~Hz})$, 121.7, 112.8, 52.8; ${ }^{19}$ F NMR (376 MHz, $\left.\mathrm{CDCl}_{3}\right) \delta-58.6$; IR (film, cm ${ }^{-1}$ ): 2961, 1733, 1659, 1565, 1498, 1305, 1261, 1151, 1132, 1115, 1014, 989, 958, 882, 784, 703; HRMS (ESI) $\mathrm{m} / \mathrm{z}$ calcd for $\left[\mathrm{M}+\mathrm{Na}^{+}\right] \mathrm{C}_{14} \mathrm{H}_{9} \mathrm{~F}_{3} \mathrm{NaO}_{4} 321.0345$, found 321.0352 .

Methyl 3-(thiophene-2-carbonyl)-4-(trifluoromethyl) benzoate: (2s) yellow solid (289mg, 92\%); mp: $103-104{ }^{\circ} \mathrm{C} ;{ }^{1} \mathbf{H}$ NMR $\left(400 \mathrm{MHz} \mathrm{CDCl}_{3}\right) \delta 8.28$ (ddd, $\left.J=8.2,1.6,0.8 \mathrm{~Hz}, 1 \mathrm{H}\right)$, $8.23-8.15(\mathrm{~m}, 1 \mathrm{H}), 7.88(\mathrm{~d}, J=8.2 \mathrm{~Hz}, 1 \mathrm{H}), 7.81$ (dd, $J=4.9$, $1.2 \mathrm{~Hz}, 1 \mathrm{H}$ ), 7.34 (dd, $J=3.8,1.2 \mathrm{~Hz}, 1 \mathrm{H}$ ), 7.14 (dd, $J=4.9,3.8$ $\mathrm{Hz}, 1 \mathrm{H}), 3.97(\mathrm{~s}, 3 \mathrm{H}) ;{ }^{13} \mathrm{C}$ NMR $\left(100 \mathrm{MHz}, \mathrm{CDCl}_{3}\right) \delta 186.3,165.0$, $143.3,138.2(\mathrm{q}, J=2.0 \mathrm{~Hz}), 136.2,136.1,133.1,131.7$ (q, $J=$ $32.8 \mathrm{~Hz}$ ), 131.0, 129.2, 128.4, $127.2(\mathrm{q}, J=4.6 \mathrm{~Hz}), 123.0(\mathrm{q}, J=$ $274.6 \mathrm{~Hz}$ ), 52.8; ${ }^{19} \mathrm{~F}$ NMR (376 MHz, $\mathrm{CDCl}_{3}$ ) $\delta$-58.5; IR (film, $\mathrm{cm}^{-1}$ ): 2957, 1734, 1643, 1513, 1441, 1411, 1304. 1277, 1192, 1139, 1116, 972, 771, 729, 681; HRMS (ESI) $\mathrm{m} / \mathrm{z}$ calcd for $\left[\mathrm{M}+\mathrm{Li}^{+}\right] \mathrm{C}_{14} \mathrm{H}_{9} \mathrm{~F}_{3} \mathrm{LiO}_{3} \mathrm{~S} 321.0379$, found 321.0389.

Methyl 3-(2-naphthoyl)-4-(trifluoromethyl) benzoate (2t): yellow solid (340mg, 95\%); mp: $133-134{ }^{\circ} \mathrm{C} ;{ }^{1} \mathbf{H}$ NMR (400 MHz, $\left.\mathrm{CDCl}_{3}\right) \delta 8.20(\mathrm{ddd}, J=8.2,1.6,0.8 \mathrm{~Hz}, 1 \mathrm{H}), 8.03(\mathrm{~d}, J=0.6 \mathrm{~Hz}$, $1 \mathrm{H}), 7.98(\mathrm{~s}, 1 \mathrm{H}), 7.91(\mathrm{dd}, J=8.6,1.7 \mathrm{~Hz}, 1 \mathrm{H}), 7.87-7.69(\mathrm{~m}$ $4 \mathrm{H}$ ), 7.52 (ddd, $J=8.2,6.9,1.2 \mathrm{~Hz}, 1 \mathrm{H}$ ), 7.42 (ddd, $J=8.1,6.9$, $1.2 \mathrm{~Hz}, 1 \mathrm{H}), 3.83(\mathrm{~s}, 3 \mathrm{H}) ;{ }^{13} \mathrm{C}$ NMR $\left(100 \mathrm{MHz}, \mathrm{CDCl}_{3}\right) \delta 194.4$, $165.1,138.9$ (q, $J=1.9 \mathrm{~Hz}$ ), 136.1, 133.5, 133.4, 133.2, 132.2, $132.0(q, J=32.7 \mathrm{~Hz}), 130.8,129.8,129.2,129.2,128.8,127.9$, 127.2 (q, $J=4.6 \mathrm{~Hz}$ ), $127.1,124.5,123.2$ (q, $J=274.6 \mathrm{~Hz}) .52 .7$; ${ }^{19}$ F NMR (376 MHz, CDCl $) \delta$-58.4. IR (film, cm ${ }^{-1}$ ): 2977, 1735, 1669, 1436, 1280, 985; HRMS (ESI) $\mathrm{m} / \mathrm{z}$ calcd for $\left[\mathrm{M}+\mathrm{Li}^{+}\right]$ $\mathrm{C}_{20} \mathrm{H}_{13} \mathrm{~F}_{3} \mathrm{LiO}_{3}$ 365.0972, found 365.0976.

Methyl 3-acetyl-4-(trifluoromethyl) benzoate (2u) : colorless oil (184mg 74\%); ${ }^{1}$ H NMR (400 MHz, $\mathrm{CDCl}_{3}$ ) $\delta 8.18$ (ddd, $J=8.2$, 1.6, $0.8 \mathrm{~Hz}, 1 \mathrm{H}), 8.13-8.06(\mathrm{~m}, 1 \mathrm{H}), 7.78(\mathrm{~d}, J=8.2 \mathrm{~Hz}, 1 \mathrm{H})$, $3.95(\mathrm{~s}, 3 \mathrm{H}), 2.60(\mathrm{~s}, 3 \mathrm{H}) ;{ }^{13} \mathrm{C}$ NMR $\left(100 \mathrm{MHz}, \mathrm{CDCl}_{3}\right) \delta 200.6$, 165.0, 140.7 (q, $J=1.9 \mathrm{~Hz}), 133.5,131.0,130.5(q, J=32.8 \mathrm{~Hz})$, 128.1, 123.1 (q, $J=274.1 \mathrm{~Hz}), 52.8,30.4(\mathrm{q}, J=1.7 \mathrm{~Hz}) ;{ }^{19} \mathbf{F}$ NMR $\left(376 \mathrm{MHz}, \mathrm{CDCl}_{3}\right) \delta-58.7$. IR (film, cm ${ }^{-1}$ ): 2958, 1730, $1711,1497,1439,1302,1263,1172,1129,1038,916,859$,
773, 748; HRMS (ESI) $\mathrm{m} / z$ calcd for $\left[\mathrm{M}+\mathrm{Li}^{+}\right] \quad \mathrm{C}_{11} \mathrm{H}_{9} \mathrm{~F}_{3} \mathrm{LiO}_{3}$ 253.0659, found 253.0667 .

Methyl 3-isobutyryl-4-(trifluoromethyl) benzoate (2v): colorless oil (159mg, 58\%); ${ }^{1} \mathbf{H}$ NMR $\left(400 \mathrm{MHz}^{\mathrm{CDCl}}{ }_{3}\right) \delta 8.28-$ $8.14(\mathrm{~m}, 1 \mathrm{H}), 8.08(\mathrm{~s}, 1 \mathrm{H}), 7.81(\mathrm{~d}, J=8.2 \mathrm{~Hz}, 1 \mathrm{H}), 3.98(\mathrm{~s}, 3 \mathrm{H})$, $3.33-3.11(\mathrm{~m}, 1 \mathrm{H}), 1.22(\mathrm{~s}, 3 \mathrm{H}), 1.20(\mathrm{~s}, 3 \mathrm{H}) ;{ }^{13} \mathrm{C}$ NMR $(100$ $\left.\mathrm{MHz}, \mathrm{CDCl}_{3}\right) \delta 207.0,165.1,140.1(\mathrm{q}, J=2.0 \mathrm{~Hz}), 131.1$ (q, $J=$ $32.6 \mathrm{~Hz}), 133.3,130.8,127.3(\mathrm{q}, J=4.9 \mathrm{~Hz}), 128.1,123.1(\mathrm{q}, J=$ $274.2 \mathrm{~Hz}), 52.7,40.4,18.0 ;{ }^{19} \mathrm{~F}$ NMR $\left(376 \mathrm{MHz} \mathrm{CDCl}_{3}\right) \delta-58.5$. IR (film, cm ${ }^{-1}$ ): 2977, 2877, 1732, 1709, 1438, 1302, 1258, 1164, 1114, 964, 918, 858, 770; HRMS (ESI) $\mathrm{m} / \mathrm{z}$ calcd for $\left[\mathrm{M}+\mathrm{Na}^{+}\right] \mathrm{C}_{13} \mathrm{H}_{13} \mathrm{~F}_{3} \mathrm{NaO}_{3}$ 297.0709, found 297.0720.

Methyl 3-benzoyl-4-(perfluoroethyl) benzoate (2w): yellow oil (255mg, 71\%); ${ }^{1} \mathrm{H}$ NMR (400 MHz, $\left.\mathrm{CDCl}_{3}\right) \delta 8.22-8.15(\mathrm{~m}, 1 \mathrm{H})$, $7.93(\mathrm{~d}, J=1.1 \mathrm{~Hz}, 1 \mathrm{H}), 7.71(\mathrm{~d}, J=8.3 \mathrm{~Hz}, 1 \mathrm{H}), 7.66(\mathrm{dd}, J=8.4$, $1.3 \mathrm{~Hz}, 2 \mathrm{H}), 7.57-7.45(\mathrm{~m}, 1 \mathrm{H}), 7.45-7.31(\mathrm{~m}, 2 \mathrm{H}), 3.84(\mathrm{~s}$, $3 \mathrm{H}) ;{ }^{13} \mathrm{C}$ NMR $\left(100 \mathrm{MHz}, \mathrm{CDCl}_{3}\right) \delta 194.1,165.0,136.1,134.0$, $133.2,130.2,130.1$ (2C), 128.9, 128.6 (2C), 52.72; ${ }^{19} \mathrm{~F}$ NMR $\left(376 \mathrm{MHz}, \mathrm{CDCl}_{3}\right) \delta-83.48,-108.18$. IR (film, $\left.\mathrm{cm}^{-1}\right)$ : 2957, 1731, 1682, 1450, 1438, 1206, 1163, 1148, 1114, 1080; HRMS (ESI) $\mathrm{m} / z$ calcd for $\left[\mathrm{M}+\mathrm{Na}^{+}\right] \mathrm{C}_{17} \mathrm{H}_{11} \mathrm{~F}_{5} \mathrm{NaO}_{3}$ 381.0521, found 381.0531.

Procedure for the gram scale synthesis of compound $\mathbf{2 b}$

In a $25 \mathrm{~mL}$ round-bottom flask equipped with magnetic stir bar, Methyl coumalate (1g, $6.5 \mathrm{mmol}, 1$ equiv), 4,4,4-trifluoro1-(p-tolyl)butane-1,3-dione 1b (1.49g, $6.5 \mathrm{mmol}, 1$ equiv) and tBuOK ( $73 \mathrm{mg}, 6.5 \mathrm{mmol}, 0.1$ equiv ) were added, and the mixture was stirred at $80{ }^{\circ} \mathrm{C}$ for 2 hours. When the reaction was completed $10 \mathrm{ml}$ absolute EtOH was added and heated to reflex to $5 \mathrm{~min}$, the mixture was cool down to room temperature and gave $1.87 \mathrm{~g}(89 \%)$ of recrystallized $\mathbf{2 b}$.

\section{Procedure for the one-pot synthesis of compound 3a}

To an oven-dried sealed tube equipped with magnetic stir bar was added methyl coumalate ( $31 \mathrm{mg}, 0.2 \mathrm{mmol}, 1$ equiv), 1a (43 $\mathrm{mg}, 0.2 \mathrm{mmol}, 1$ equiv) and $t \mathrm{BuOK}(2 \mathrm{mg}, 0.2 \mathrm{mmol}, 0.1$ equiv). The reaction was stirred at $80^{\circ} \mathrm{C}$ for 2 hours, cool to rt then $\mathrm{Pd}(\mathrm{OAc})_{2}$ ( $4.5 \mathrm{mg}, 0.02 \mathrm{mmol}, 0.1$ equiv) followed by $\mathrm{Ag}_{2} \mathrm{O}$ ( $69 \mathrm{mg}, 0.3 \mathrm{mmol}, 1.5$ equiv) were added and the flask purged three times with argon. Trifluoroacetic acid $(2 \mathrm{~mL})$ was added to the system and the reaction mixture was stirred at $130{ }^{\circ} \mathrm{C}$ for $36 \mathrm{~h}$. After completion of the reaction, the reaction mixture was cooled, filtered through a short pad of Celite ${ }^{\circledR}$ and washed several times with dichloromethane. The combined filtrate was concentrated under vacuum and the residue purified by flash chromatography on silica gel column using cyclohexane/EtOAc as eluent to give the desired product 3a.

Methyl 9-oxo-1- (trifluoromethyl) -9H- fluorene-4-carboxylate (3a): yellow solid (43.4 mg, 66\%); ${ }^{1} \mathbf{H}$ NMR $\left(400 \mathrm{MHz} \mathrm{CDCl}_{3}\right) \delta$ $8.09(\mathrm{dt}, J=7.8,0.8 \mathrm{~Hz}, 1 \mathrm{H}), 7.95(\mathrm{dd}, J=8.2,0.7 \mathrm{~Hz}, 1 \mathrm{H}), 7.75$ (ddd, $J=7.4,1.3,0.7 \mathrm{~Hz}, 1 \mathrm{H}), 7.62(\mathrm{~d}, J=8.2 \mathrm{~Hz}, 1 \mathrm{H}), 7.54(\mathrm{td}, J$ $=7.7,1.3 \mathrm{~Hz}, 1 \mathrm{H}), 7.41(\mathrm{td}, J=7.4,0.8 \mathrm{~Hz}, 1 \mathrm{H}), 4.05(\mathrm{~s}, 3 \mathrm{H}) ;{ }^{13} \mathrm{C}$ NMR $\left(100 \mathrm{MHz} \mathrm{CDCl}_{3}\right) \delta 189.0,166.6,145.4,141.4,135.3$, $135.3,133.7,132.5,130.6,130.2$ (q, $J=35.4 \mathrm{~Hz}$ ), 130.1, 125.7 (q, $J=5.9 \mathrm{~Hz}), 125.4,124.6,122.1(\mathrm{q}, J=274.6 \mathrm{~Hz}), 53.0 ;{ }^{19} \mathrm{~F}$ NMR (376 MHz, $\mathrm{CDCl}_{3}$ ) $\delta-61.7$; HRMS (ESI) $\mathrm{m} / \mathrm{z}$ calcd for $\left[\mathrm{M}+\mathrm{Na}^{+}\right] \mathrm{C}_{16} \mathrm{H}_{9} \mathrm{~F}_{3} \mathrm{NaO}_{3} 329.0396$ found 329.0397 . 


\section{Conflicts of interest}

The authors declare no competing financial interest.

\section{Acknowledgements}

We thank the University P. et M. Curie (UPMC) and CNRS for funding. The Fédération de Recherche (FR2769) provided technical access for analysis. L.C. thanks the China Scholarship Council (CSC) for a PhD grant. N. K., a master student at Ludwig Maximilian University of Munich, was a 2017 Erasmus+ trainee.

\section{Notes and references}

1 For their impact in organic and medicinal chemistry see $(a) \mathrm{R}$. Filler, Y. Kobayashi, L. M. Yagupolskii, Organofluorine Compounds in Medicinal Chemistry and Biomedical Applications Elsevier, Amsterdam, 1993. (b) T. Hiyama, Organofluorine Compounds, Chemistry and Applications, Springer-Verlag, Berlin, 2000. (c) R.D. Chambers, Fluorine in Organic Chemistry, Blackwell Publishing/CRC Press, Boca Raton, 2004. (d) W. K. Hagmann, J. Med. Chem. 2008, 51 4359. (e) N. A. Meanwell, J. Med. Chem. 2011, 54, 2529. (f) P Kirsch, Modern Fluoroorganic Chemistry. VCH, Weinheim, 2013. (g) J. Wang, M. Sanchez-Rosello, J. L. Acena, C. de Pozo, A. E. Sorochinsky, S. Fustero, V. A. Soloshonok, H. Liu, Chem. Rev. 2014, 114, 2432.

2 For a review see: S. Purser, P. R. Moore, S. Swallow, V. Gouverneur, Chem. Soc. Rev. 2008, 37, 320.

3 (a) X. Wang, L. Truesdale. J. -Q. Yu, J. Am. Chem. Soc., 2010 132, 3648. (b) X. Zhang, H. Dai, M. Wasa, J.-Q Yu. J. Am. Chem. Soc., 2012, 134, 11948. (c) L. Hu, X. Chen, Q. Gui, Z. Tan, G. Zhu, Chem. Commun., 2016, 52, 6845. (d) P. Liu, W. Liu, C.-J. Li, J. Am. Chem. Soc., 2017, 139, 14315.

4 (a) H. Morimoto, T. Tsubogo, N. D. Litvinas, J. F. Hartwig, Angew. Chem. Int. Ed. 2011, 50, 3793. (b) Z. Weng, R. Lee, W. Jia, Y. Yuan, W. Wang, X. Feng, K-W. Huang, Organometallics, 2011, 30, 3229. (c) L. Chu, F-L. Qing, Org. Lett. 2010, 12, 5060. (d) T. D. Senecal, A.T. Parsons, S. L. Buchwald, J. Org. Chem. 2011, 76, 1174. (e) S. L. Clarke, G. P. McGlacken, Chem. Eur. J. 2017, 23, 1219.

5 (a) H. Urata, T. Fuchikami, Tetrahedron Lett. 1991, 32, 91. (b) G. G. Dubinina, H. Furutachi, D. A. Vicic, J. Am. Chem. Soc. 2008, 130, 8600. (c) A. Hafner, S. Bräse, Angew. Chem. Int. Ed. 2012, 51, 3713.

6 F. Sladojevich, E. McNeill, J. Börgel, S-L. Zheng, T. Ritter, Angew. Chem. Int. Ed. 2015, 54, 3712.

7 (a) I. Kieltsch, P. Eisenberger, A. Togni, Angew. Chem. Int. Ed. 2007, 46, 754. (b) V. Matousek, E. Pietrasiak, R. Schwenk, A. Togni, J. Org. Chem. 2013, 78, 6763. (c) Y. Kuninobu, M Nishi, M. Kanai, Org. Biomol. Chem. 2016, 14, 8092.

8 For trifluoromethylation by photoredox catalysis see: D. A. Nagib, D. MacMillan, Nature, 2011, 480, 224.

9 (a)T. Konno, K. Moriyasu, R. Kinugawa, T. Ishihara, Org. Biomol. Chem. 2010, 8, 1718. (b) Y. Sakaguchi, Y. Nakazaki, K. Moriyasu, T. Konno, J. Fluor. Chem. 2015, 179, 64.

10 For formal [4+2] CA see: (a) J-N. Volle, M. Schlosser, Eur. J. Org. Chem. 2002, 9, 1490. (b) D. M. Volochnyuk, A. N Kostyuk, D. A. Sibgatulin, A. N. Chernega, A.M. Pinchuk, A. Tolmachev, Tetrahedron 2004, 60, 2361. (c) V. O. laroshenko, A. Bunescu, L. Domke, A. Spannenberg, D. V. Sevenard, A. Villinger, V. Sosnovskikh, P. Langer, J. Fluor. Chem. 2011, 132 441. For formal [3+3] see: (d) C. Mamat, T. Pundt, A. Schmidt, P. Langer,. Tetrahedron Lett. 2006, 47, 2183. (e) C. Mamat, T. Pundt, T. Tam Dang, R. Klassen, H. Reinke, M. Köckerling, P. Langer, Eur. J. Org. Chem. 2008, 3, 492.
11 For reviews see: $(a)$ K. Afarinkia, V. Vinader, T. Nelson, G. H. Posner, Tetrahedron, 1992, 48, 9111. (b) A. Goel, V. J. Ram, Tetrahedron, 2009, 65, 7865.

12 (a) J. D. Kirkham, A. G. Leach, E. C. Row, J. Harrity. Synthesis, 2012, 44, 1964. (b) I. S. Kondratov, N. A. Tolmachova, V. Dolovanyuk, I. Gerus, K. Bergander, C. G. Daniliuc, G. Haufe, Eur. J. Org. Chem. 2014, 12, 2443. (c) I. S. Kondratov, N. A. Tolmachova, V. G. Dolovanyuk, I. I. Gerus, C-G. Daniliuc, G. Haufe, Eur. J. Org. Chem. 2015, 11, 2482.

13 G. A. Kraus, S Riley, T. Cordes, Green Chem. 2011, 13, 2734.

14 (a) T. Pfennig, J. M. Carraher, A. Chemburkar, R. L. Johnson, A. T. Anderson, J. P. Tessonnier, M. Neurock, B. H. Shanks, Green Chem., 2017, 19, 4879. (b) T. Pfennig, R. L. Johnson, B. H. Shanks, Green Chem., 2017, 19, 3263.

15 (a) M. Haji Abdullahi, L. M. Thompson, M. J. Bearpark, V. Vinader, K. Afarinkia, Tetrahedron, 2016, 72, 6021. (b) J. J. Lee, G. A. Kraus, Tetrahedron Lett. 2013, 54, 2366. (c) J. J. Lee, G. R. Pollock III, D. Mitchell, L. Kasuga, G. A. Kraus, RSC Advances, 2014, 4, 45657. (d) G. A. Kraus, G. R. Pollock III, C. L. Beck, K. Palmer, A. H. Winter, RSC. Adv. 2013, 3, 12721. (e) J. J. Lee, G. A. Kraus, Green Chem. 2014, 16, 2111. (f) T. Guney, J. J. Lee, G. A. Kraus, Org. Lett. 2014, 16, 1124. (g) L. R. Domingo, J. A. Saez, S. R. Emamian, Org. Biomol. Chem. 2015, 13, 2034.

16 T. J. Greshock, R. L. Funk, J. Am. Chem. Soc. 2006, 128, 4946.

17 R. L. Greenaway, C. D. Campbell, O. T. Holton, C. A. Russell, E. A. Anderson, Chem. Eur. J. 2011, 17, 14366.

18 J. Deng, W. Chen, C. Zhu, S. Chuang, Adv. Synth. Catal. 2015, $357,1453$.

19 X. Li, H. Yu, Y. Huang, Adv. Synth. Catal. 2017, 359, 1379.

20 (a) J. Agarwal, O. Bayounes, S. Thorimbert, L. Dechoux, RSC Advances, 2014, 4, 2772. (b) K. Plevovà, L. Chang, E. Martin, Q. Llopis, L. Dechoux, S. Thorimbert, Adv. Synth. Catal. 2016, 358, 3293. (c) L. Chang, K. Plevovà, S. Thorimbert, L. Dechoux, J. Org. Chem, 2017, 82, 5499.

21 S. Büttner, A. Riahi, I. Hussain, M. A. Yawer, M. Lubbe, A. Villinger, H. Reinke, C. Fischer, P. Langer, Tetrahedron, 2009, 65, 2124.

22 M. Mamat, H. P. Reinke, P. Langer, Naturforsch. 2009, 64b, 423.

23 T. Q. Yu, Y. Fu, L. Liu, Q. X. Guo J. Org. Chem., 2006, 71, 6157.

24 (a) H. Li, R. Zhu, W. Shi, K. He, Z. Shi, Org. Lett., 2012, 14 , 4850. (b) P. Gandeepan, C.H. Hung, C. H. Cheng, Chem. Commun., 2012, 48, 9379.

25 L. M. Bishop, J. E. Barbarow, R. E. Bergman, D. Trauner Angew. Chem. Int. Ed. 2008, 47, 8100.

26 L. M. Bishop, R. E. Roberson, R. G. Bergman, D. Trauner, Synthesis, 2010, 2233.

27 N. A. Magomedov, P. L. Ruggiero, Y. Tang, J. Am. Chem. Soc. 2004, 126, 1624

28 (a) O. Doebner, Ber. Dtsch. Chem. Ges. 1902, 25, 1136. (b) A. Galat, J. Am. Chem. Soc. 1946, 68, 376. (c) S. Thorimbert, C. Taillier, S. Bareyt, D. Humilière, M. Malacria, Tetrahedron Lett. 2004, 45, 9123. (d) A. Ear, V. Toum, S. Thorimbert, L. Dechoux Synlett 2014, 25, 1713.

29 For a review concerning the bioactivities of natural benzophenones see: S-B. Wu, C. Long, E. J. Kennelly, Nat. Prod. Rep. 2014, 31, 1158.

30 For some publications concerning biological effects of fluorinated benzophenone derivatives see: (a) J. H. Chan, G A. Freeman, J. H. Tidwell, K. R. Romines, L. T. Schaller, J. R Cowan, S. S. Gonzales, G. S. Lowell, C. W. Andrews III, D. J. Reynolds, M. St Clair, R. J. Hazen, R. G. Ferris, K. L. Creech, G. B. Roberts, S. A. Short, K. Weaver, G. W. Koszalka, L. R. Boone, J. Med. Chem. 2004, 47, 1175. (b) J. Ren, P. P. Chamberlain, A. Stamp, S. A. Short, K. L. Weaver, K. R. Romines, R. Hazen, A. Freeman, R. G. Ferris, C. Webster Andrews, L. Boone, J. H. Chan, D. K. Stammers, J. Med. Chem. 2008, 51, 5000. (c) F. Belluti, A. De Simone, A. Tarozzi, M. 
Bartolini, A. Djemil, A. Bisi, S. Gobbi, S. Montanari, A. Cavalli, V. Andrisano, G. Bottegoni, A. Rampa, Eur. J. Med. Chem. 2014, 78, 157. 